\title{
Attitude to health among athletes with extremities injuries
}

\author{
Lyubov Malikova*, Natalia Yakovleva \\ Ryazan State Medical University named after academician I.P. Pavlov, 390026, Ryazan, Russia
}

\begin{abstract}
Attitude to health presents the inner mechanism of activity and behavior self-regulation. For a professional athlete positive attitude to health is the base for a successful career. However, the system of a personality's attitudes is a dynamic construct. It is influenced by many factors, including sports injuries. During the research we tried to describe the peculiarities of health-protecting behavior, including the attitude to health of professional athletes with extremities injuries. In order to define the specific influence of sport as a professional activity we chose athletesamateurs with extremities injuries as the group of comparison. The results of the held research prove that the attitude to health is the main determinant of professional athletes health-protecting behavior and at the same time, it is not included into the model of health -protection of athletes- amateurs. It reflects the influence of professional specificity in the system of personality's attitudes formation.
\end{abstract}

\section{Introduction}

The concept of a personality's attitudes is developed in the works of V.N. Myasishchev, A.F. Lazurskiy, B.G. Ananev, B.F. Lomov [1]. In the opinion of V.N. Myasishchev, psychic attitudes reflect the connection of a person with the reality. "...it characterizes the personality as an active subject with his selective character of inner emotions and outer actions, directed toward different sides of an objective reality" [1, p. 101]. It means that psychic attitudes form the inner mechanism of a person's activity and behavior selfregulation. That is why the attitude to health can be considered as the target of psychiccorrectional influence of a specialist, who works in the sphere of health psychology [1]. The attitude to health was firstly considered by D.N. Loranskiy. The scientists, who work in this direction (G.S. Nikiforov, T.V. Belinskaya), include three components into the structure of "attitude to health" construct: cognitive, emotional and behavioral. R.A. Berezovskaya mentions the necessity to include the separate axiological-motivational component of the attitude to health, as the connecting one between cognitive and behavioral parts [1].

At the same time, the attitude to health is not an isolated part of the personality's attitudes system, which influences the whole life of a person and first of all the peculiarities of health-protecting behavior $[3,4]$. Construct of health-protecting behavior includes

* Corresponding author: malikovala@yandex.ru 
functional and structural parts. A functional concept of health-protecting behavior is presented by the peculiarities of health-protecting activity (V.A. Uryvaev, (2012), N.V. Yakovleva (2015); by "Ilness career" notion (G. Huppmann, F. Wilker (1988) and by the behavior in the situation of health level decrease (M.M. Kabanov, V.M. Smirnov). Structural component corresponds with the structural component of inner health state (V.E. Kagan, N.N. Vishnyakov, E.I. Pervichko) [3].

The attitude to health is the part of health-protecting behavior, however, the role of this factor can vary depending on age, sphere of activity, the definite situation of life.

Professional sport, which is characterized by high physical and psychological loads, restrictions and stress makes this kind of activity close to extreme one [5]. Moreover, the sport of higher achievements is connected with injuries. That is why health for a professional athlete is the most important terminal value. It helps to achieve professional realization. Health violation in the situation of sports injury concerns all levels of an individual's functioning, from perceptional to axiological-notional $[4,6]$. The degree of behavior self-regulation is determined by the peculiarities of the attitude to health in such a difficult for an athlete situation as injury, defines the peculiarities of rehabilitation process [7].

Taking into account the theoretical base and experts' opinion of sports doctors, we supposed that the attitude to health forms an important determinant of health-protecting behavior of a professional athlete.

\section{Materials and Methods}

An empiric research was held on the basis of state public establishment of health "Ryazan regional medical-physical culture dispensary" and state budgetary establishment of Ryazan region "Regional clinical hospital" (traumatology department).

In general 62 male athletes took part in the research. The average age of the respondents was $22 \pm 3,54$. The athletes were the representatives of such kinds of sport as hockey, football, volleyball, athletics, judo, sambo, boxing.

In accordance with the aim and hypothesis of the research we formed the main group and the comparison group.

The main group includes professional athletes, who have extremities injuries (32 respondents, the average age $21,75 \pm 3,24)$. The comparison group included athletesamateurs with extremities injuries (30 respondents, the average age $22 \pm 3,89$ ).

The respondents in the groups didn't differ in injury localization, all athletes had lower extremities injuries of the same severity level (macro-injuries: sprain, bursting, muscles and joints rupture, dislocation).

In accordance with the defined construct in order to describe the model of healthprotection we used the following standardized methodologies: "Index of attitude to health" (S. Deryabo, V. Yasvin, 1999); "Questionnaire of health-protecting activity study" (Yakovleva N.V., Yakovlev V.V., 2012); "Numerical Rating Scale, NRS” (McCaffery M., Beebe A. 1993); "Toronto Alexithymia Scale" (TAS-20-R) modified in 1994 by G. Taylor with other authors (adaptattion: G. Starostina, G. D. Taylor, L. K. Kvilti, A. E. Bobrov); "The level of subjective control" (E. F. Bazhin, E. A. Golynkina, A. M. Etkind, 1984). Mathematical-statistical handling was held with the help of nonparametric Mann Whitney U-criterion, correlational analysis, factorial analysis (principal components method, varimax- rotation).

\section{Results and Discussion}


The first stage of our research was studying the differences validity using nonparametric Mann Whitney U-criterion (in terms of $p<0,01$ ) in the groups. It was revealed that there were differences according to the intensity of the attitude emotional component to health. The indices of professional athletes $(\mathrm{Mx}=5,4)$ were considerably higher, than in the group of comparison $(\mathrm{Mx}=3,5)$. Such kind of peculiarity demonstrates that for professional athletes it is more typical to have feelings and emotions connected with health state and its change. The attitude to health among athletes-amateurs almost doesn't influence emotional sphere and very often a healthy life style is connected with negative emotional stereotypes. In spite of the fact that the indices of this scale among professional athletes are average, which can be connected with the injury, emotional component turned out to be more important for them.

We received valid differences of the attitude behavioral sphere to health in the examined groups $(\mathrm{Mx}=6,6$ and $\mathrm{Mx}=5,3)$. The actions directed toward care for health state and realized in practical activity, in a form of training lessons, health-improving procedures, both personally and under the influence of a coach, are typical for professional athletes, which seems logical.

Moreover, motivational-axiological sphere of health-protection among professional athletes $(\mathrm{Mx}=6,34)$ differs considerably from motivational-axiological sphere of athletesamateurs $(\mathrm{Mx}=5,2)$ ("Stusying health-protecting activity" methodology). The desire to go in for sports among professionals is conditioned by specific motives and values. They are more directed toward a healthy life style, owing to the need for self-actualization realization in profession [3].

At the same time, both professionals and amateurs know the ways of health improvement, have the desire to get new information concerning the ways of health improvement, the ways of these or that exercises and the techniques fulfillment. In general the indices of attitude level to own health state among professional athletes are considerably higher. It shows the involvement of the attitude to health state and its parts into healthprotecting behavior and health-protection regulation.

The next stage was the interconnections study between the components of the attitude to health and the components of health-protection.

Emotional sphere of the attitudes to health system is connected with the motivation $\left(\mathrm{r}=0,39^{*}\right.$, here and further: ${ }^{*}-$ in terms of $\left.\mathrm{p}<0,05, * *-\mathrm{p}<0,01\right)$ and self-effectiveness $\left(\mathrm{r}=-0,42^{*}\right)$ of health-protection. Excessive emotional experience concerning own health state is connected with motives formation and actualization. They are directed toward favorable state of an organism support and at the same time, with a low estimation of own activity effectiveness in terms of health.

Cognitive component of the attitude to health system among athletes is also connected with motivational sphere $\left(\mathrm{r}=0,49^{* *}\right)$ and goal-setting $\left(\mathrm{r}=0,59^{* *}\right)$. This peculiarity is demonstrated in a fact that searching for information concerning the problems of a healthy life style and health improvement, communication with competent specialists, is connected with motives and values of health-protection formation, the ability to set the goals in order to improve a health sate.

Behavioral component of professionals' attitude to own health state is also connected with the peculiarities of goal-setting $(\mathrm{r}=0,502 * *)$. Fulfilling the actions connected with own health improvement (leading healthy life style, special exercises, health-improving procedures fulfillment, corresponding abilities and skills formation) is connected with own sports activity realization in general and the aims selection for the result achievement, the definite exercises and the ways of health support selection. It would help to achieve the desired result. 
We also revealed correlation between the components of the attitude to health state and the intensity of pain $\left(\mathrm{r}=-0,48^{* *}\right)[6,8]$, internal personal orientation in the sphere of health and sickness $\left(\mathrm{r}=0,47^{* *}\right)$, internality in the sphere of achievements $(\mathrm{r}=0,44 *)$.

The distinctive feature of health-protection among athletes-amateurs is the absence of interconnections of the attitude to health behavioral component and other elements of health-protection, in contrast to highly-qualified athletes. Emotional sphere is connected with alexithimical features, in particular with the difficulties of feelings description $\left(\mathrm{r}=0,62^{* *}\right)$, and with the intensity of pain senses $\left(\mathrm{r}=-0,4^{*}\right)$. It should be noted that the attitude to health state in the group of comparison is connected with the standards of health. It proves outer conditioned character of the standard notions of health and the ways of its preservation, in contrast to highly-qualified athletes, among whom these notions are internally formed and independent of the system of attitudes to health.

A.V. Karpov and N.V. Yakovleva in their work about the models of health among athletes-professionals and amateurs mention that an individual model of athletes' health is specific owing to sign system of health estimation. It is typical for professional athletes. The authors suppose that such specificity of standard-criteria system of health estimation is conditioned by health importance for a successful professional career [9].

The received results reflect the definite interconnections and don't give precise understanding of the role, which the attitude to health plays in health-protecting behavior. For this purpose we held factorial analysis (principal components method, varimaxrotation). In the group of professional athletes the first factor, which describes $22,61 \%$ of the whole variance of variables, includes the following scales of "Index of the attitude to health" methodology: "General intensity in terms of health", "Actions based scale", "Cognitive scale", "Emotional scale". This factor is called by us "Attitude to health". General index of the attitude to health state is more important in this factor, as it reflects the importance of the formed attitude to health for a professional athlete. The developed and positive attitude to health conditions health-protecting behavior actualization, patterns of a healthy life style formation. It then provide success in professional sport. Less important is the variable. It reflects the attitude to health in the sphere of the fulfilled actions of an athlete - behavioral component of the attitude to health. The third variable in the structure of this factor is cognitive activity. It is important for an athlete not only to estimate health state independently, but also get an objective competent information from the specialists and search for additional information in different sources: mass media, Internet, magazines, newspapers, books. The considered factor also includes emotional attitude to health, reflecting emotional experience, connected with health state and injury overcoming.

Revealed by us factor demonstrates the inclusion of all three components of the attitude to health structure: cognitive, emotional and behavioral. The structure of the professional athletes' attitude system has specificity in a form of great importance of behavioral component. We suppose that behavioral component becomes especially urgent in the situation of an injury, when an athlete has to fulfill the definite rehabilitation exercises. The involvement into activity helps an athlete to be less worried about the process of rehabilitation and the feelings connected with the injury become less staticized. Apart from "Attitude to health" factor, factorial model of health-protection of professional athletes includes 6 factors. They in general explain 71,09\% of variance of the corresponding variables.

The model of health-protection among athletes-amateurs also includes 7 factors. They explain $81 \%$ of variables dispersion. In contrast to the model of highly-qualified athletes, in the model of amateurs dominant position takes the factor, presented by the scales of "The level of subjective control" methodology. As the average results according to these scales demonstrate high level of externality among athletes-amateurs, this factor was called by us 
"Externality". It is interesting that none of the variables, which reflect the index of the attitude to health, was reflected in the model of health-protection of athletes-amateurs. Such a peculiarity makes us come to the conclusion that health-protecting behavior of athletesamateurs is conditioned by other determinants and the peculiarities of their attitude to health state don't regulate behavior, directed toward health preservation.

Thus, the attitude to health among professional athletes is conditioned by specific motives, feelings and emotions, connected with health state and peculiarities of behavior in the sphere of health.

\section{Conclusions}

The inclusion of sport into professional activity conditions highly-qualified athlete's specific system of attitudes formation. In terms of an injury the attitude to health forms the main determinant and regulator of health-protecting behavior.

Among the components of the attitudes to health system the first place takes behavioral component, athlete's involvement into the process of rehabilitation after an injury.

Attitude to health is not reflected in the model of athletes-amateurs health-protecting behavior, which shows us professional specificity of highly-qualified athletes attitudes to health system [9].

At the same time, the components of the attitude to health in the group of athletesamateurs are connected with motivational-axiological sphere of health-protection and the peculiarities of goal-setting, as among professionals. However, for athletes-amateurs it is more typical to take into account outer standards of health, have difficulties in understanding feelings connected with health.

\section{References}

1. R.A. Berezovskaya, Sain-Petersburg State University bulletin, 1 (2011).

2. V.N. Myasishchev, Psychology of realtions (2004)

3. G.A. Arina, M.A. Iosifyan, V.V. Nikolaeva, National Psychological Journal, 4 (2018).

4. A.V. Eganov, A.M. Kuzmin, S.S. Korovin, V.S. Bykov, Theory and Practice of Physical Culture, 5 (2016)

5. L.M. Dovzhik, K.A. Bochaver, Psychology of sports injury Sport (2020)

6. L.A. Malikova, N.V. Yakovleva, Russian Journal of Physical Education and Sport, 15(2), 99-107 (2020)

7. D. K. Chan, M. S. Hagger, J Sci Med Sport, 15 (2012)

8. J.S. Everhart, A.J. Chafitz, K.M. Harris, S.E. Schiele, C.F. Emery, D.C. Flanigan, Journal of Science and Medicine in Sport, 23 (2020)

9. N.V. Yakovleva, A.V. Karpov, Kostroma State University bulletin. Series: Pedagogics. Psychology. Socialkinetics, 20 (2014) 